\title{
Brain Abscess Due to Nocardia in a Patient With Systemic Lupus Erythematosus
}

Zhuoxuan Li, BM, Dongling Li, BM, Pan Lv, BM, Jianping Liu, Professor, Department of Rheumatology and Immunology, Affiliated Hospital of North Sichuan Medical College, Nanchong, China. Address correspondence to Prof. J. Liu, Department of Rheumatology and Immunology, Affiliated Hospital of North Sichuan Medical College, Nanchong, China, No. 1 South MaoYuan Road, Shunqing District, Nanchong 637000, China. Email: ljpbr@sina.com. This study was funded by the National Natural Science Foundation of China (81972119). The authors declare no conflicts of interest relevant to this study. In order to protect the privacy of the patient, deidentified case details and images were used. The patient was informed by phone due to the prevalence of the COVID-19 about the publication of the manuscript and we obtained patient informed consent to publish the material. According to national and local regulations, the approval by Ethics Committee is not required for a case report.

Nocardia brain abscesses mainly occur in immunosuppressive hosts $^{1}$ and comprise only $2 \%$ of all intracranial abscesses. It is difficult to identify central nervous system infections in patients with systemic lupus erythematosus because of the silent clinical manifestations and their simulation of lupus encephalopathy. ${ }^{2}$ The higher mortality rate is often the result of misdiagnosis. ${ }^{3}$ Magnetic resonance imaging (MRI) and etiological examination are helpful for a differential diagnosis.

A 52-year-old female was diagnosed with SLE 4 months ago. Her disease had initially been characterized by polyarthritis, proteinuria, butterfly erythema, dental ulcers, alopecia, and nephritis. She was treated with prednisone $80 \mathrm{mg} / \mathrm{d}$ per oral (PO), cyclophosphamide $0.6 \mathrm{mg}$ intravenous injection twice monthly (in total, 2 infusions and then treated with mycophenolate mofetil [MMF] $750 \mathrm{mg}$ PO twice daily), and hydroxychloroquine $200 \mathrm{mg}$ PO twice daily, with good response. While the prednisone dose was tapered to $25 \mathrm{mg} / \mathrm{d}$ with MMF $500 \mathrm{mg}$ twice daily, she presented to our hospital with persistent frontal-parietal headache accompanied by cognitive dysfunction for 2 days. Laboratory analysis at admission showed the following:

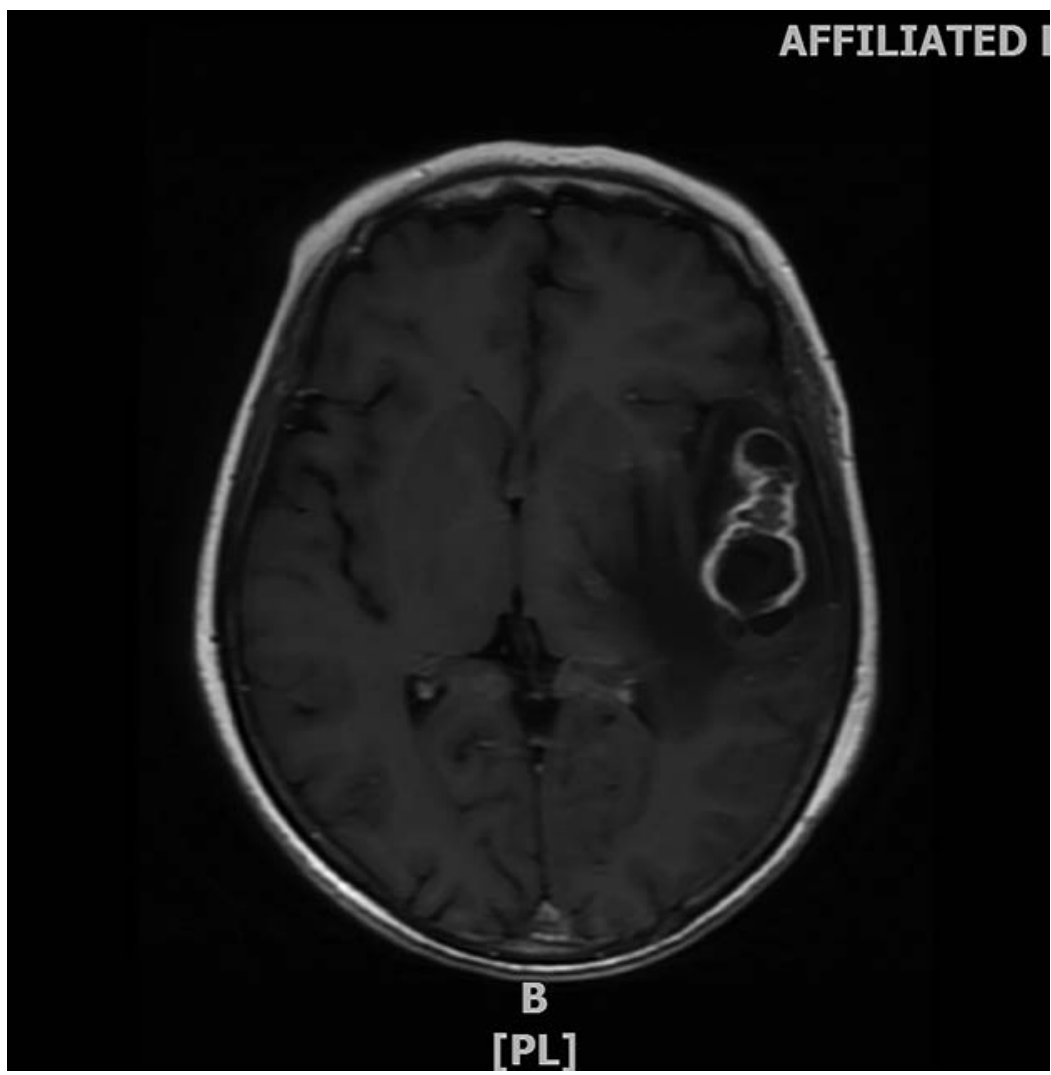

Figure 1. MRI T1-weighted postcontrast image demonstrating irregular ringlike contrast-enhanced lesions. MRI: magnetic resonance imaging. 
a leukocyte count of $7.62 \times 10^{9} / \mathrm{L}$ with neutrophil count of $80.50 \%$, and high-sensitivity C-reactive protein $(21.24 \mathrm{mg} / \mathrm{L})$. Brain MRI revealed irregular ringlike contrast-enhanced lesions in the left temporal lobe (Figure 1). Surgical brain biopsy revealed pyogenic abscesses. Contrary to acid-fast staining, weak acid-fast staining tested positive, and Gram staining revealed Gram-positive rods with branching (Figure 2). Cultures on blood agar plates produced a growth of Nocardia. We then treated her with cotrimoxazole $0.96 \mathrm{~g}$ PO 3 times daily for 45 days with linezolid $600 \mathrm{mg} / 12 \mathrm{~h}$ for 9 days. After 6 months, the patient experienced clinical and radiological improvement.

\section{REFERENCES}

1. Burke VE, Lopez FA. Approach to skin and soft tissue infections in non-HIV immunocompromised hosts. Curr Opin Infect Dis 2017;30:354-63.

2. Saltman AP, Tseng E, Bunce PE, Albert L. Double trouble: a case of concurrent opportunistic infections. J Rheumatol 2015;42:903-4.

3. Mc-Nab P, Fuentealba C, Ballesteros F, et al. [Nocardia asteroides infection in a patient with systemic lupus erythematosus.] [Article in Spanish] Rev Med Chil 2000;128:526-8.

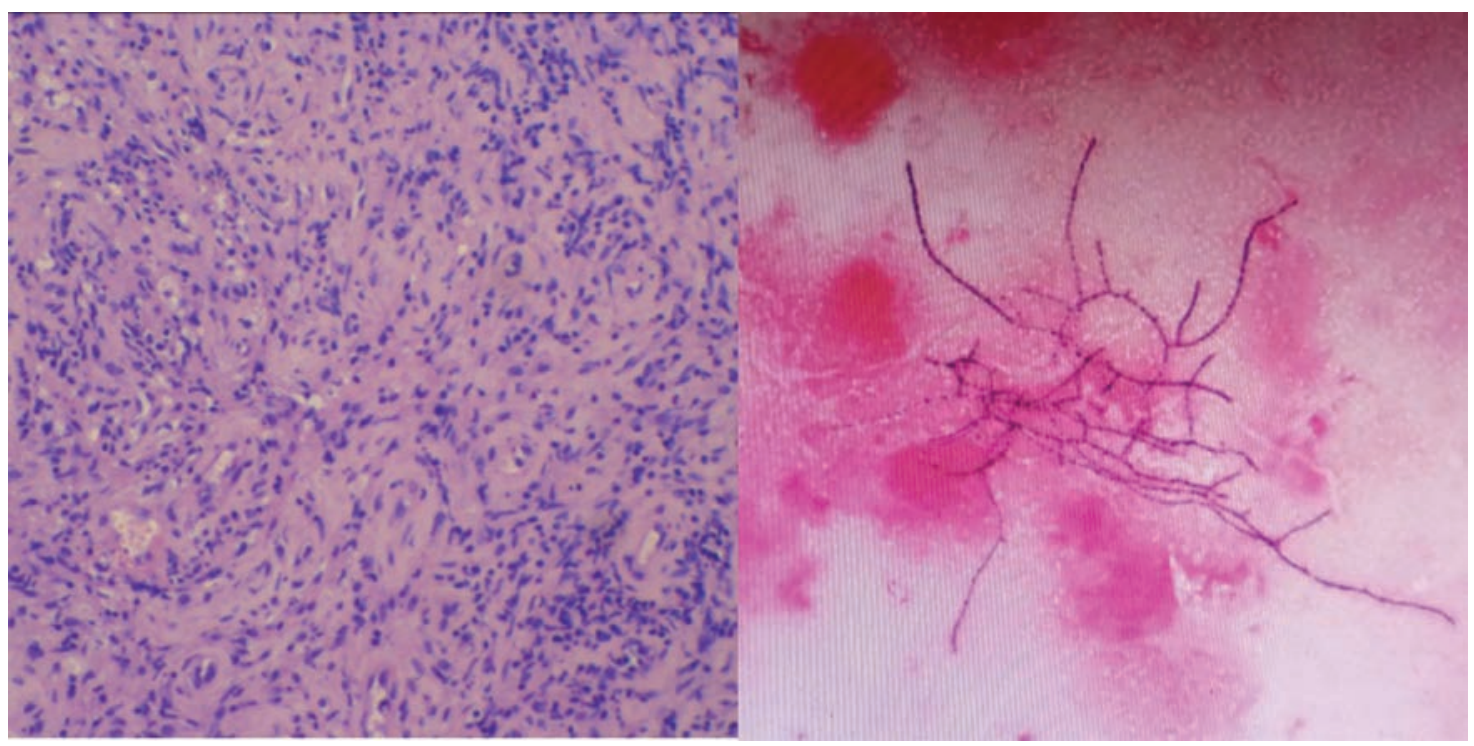

Figure 2. Brain biopsy and bacterial staining of a patient with Nocardia brain abscess. Paraffin section was predominantly infiltrated with acute and chronic inflammatory cells and showed focal inflammatory granulation tissue hyperplasia. Gram stain revealed Grampositive rods with branching. 